\title{
Impact of molecular subtypes on metastatic behavior and overall survival in patients with metastatic breast cancer: A single-center study combined with a large cohort study based on the Surveillance, Epidemiology and End Results database
}

\author{
HONG YANG $^{1,2}$, RONG WANG $^{1}$, FUREN ZENG $^{2}$, JIE ZHAO $^{3}$, SHUNLI PENG ${ }^{1}$, YUEYUN MA $^{1}$, \\ SHIYU CHEN ${ }^{1}$, SIJIE DING ${ }^{1}$, LONGHUI ZHONG ${ }^{1}$, WEI GUO ${ }^{3}$ and WEI WANG ${ }^{1}$ \\ ${ }^{1}$ Department of Radiation Oncology, Nanfang Hospital, Southern Medical University, Guangzhou, \\ Guangdong 510515; Departments of ${ }^{2}$ Oncology and ${ }^{3}$ Pathology, Hunan Provincial People's Hospital and \\ The First Affiliated Hospital of Hunan Normal University, Changsha, Hunan 410000, P.R. China
}

Received February 10, 2020; Accepted July 6, 2020

DOI: $10.3892 / \mathrm{ol} .2020 .11948$

\begin{abstract}
Breast cancer is a highly heterogeneous disease at the molecular level and $>90 \%$ of mortalities are due to metastasis and its associated complications. The present study determined the impact of molecular subtypes on metastatic behavior and overall survival (OS) of patients with metastatic breast cancer. The influence of molecular subtypes on the sites and number of metastases in 166 patients with metastatic breast cancer from a single center were assessed; and the influence of molecular subtypes on the sites and number of metastases and OS in 15,322 metastatic cases among 329,770 patients with primary breast cancer from the Surveillance, Epidemiology and End Results database were assessed. Analysis of both datasets revealed that different molecular subtypes exhibited differences in the prevalence of different metastatic sites and number of metastases. A larger proportion of bone metastasis was observed in the hormone receptor (HR)+/human epidermal growth factor receptor 2 (HER2)+ subtype than in other subtypes, more lung metastasis was observed in the HR-/HER2+ subtype and more liver metastasis occurred in the HR+/HER2+ and HR-/HER2+ subtypes. Single-site metastasis was more common for the HR+/HER2- subtype than in other subtypes, while 2-3 sites of metastases were more common for the HR+/HER2+ subtype and $\geq 4$ sites of metastases were more frequent in the HR-/HER2+ and HR-/HER2- subtypes. The mean OS of patients with primary
\end{abstract}

Correspondence to: Professor Wei Wang, Department of Radiation Oncology, Nanfang Hospital, Southern Medical University, 1838 Guangzhou N Avenue, Guangzhou, Guangdong 510515, P.R. China

E-mail: wangwei9500@hotmail.com

Key words: breast cancer, molecular subtypes, metastatic sites, number of metastatic sites, overall survival breast cancer in the HR+/HER2- subtype group was the longest (78.5 months), while the HR-/HER2- group had the shortest mean OS (69.1 months). The mean OS of the metastatic HR+/HER2+ group was the longest (46.0 months), while the mean OS of the metastatic HR-/HER2- group was the shortest (18.5 months). In conclusion, the results of the present study suggested that different molecular subtypes of breast cancer have different metastatic behavior, as well as mean OS.

\section{Introduction}

Breast cancer is the most common type of malignancy in females and $>90 \%$ of mortalities are due to metastasis-associated complications (1). An estimated 5-10\% of patients have metastasis at the time-point of diagnosis and $20-40 \%$ of those who did not have any metastasis at the time-point of diagnosis eventually experience recurrence after treatment and metastasis; once recurrence and metastasis occur, the overall prognosis is generally poor $(2,3)$.

The status of hormone receptors (HRs) is closely linked to the treatment and prognosis of breast cancer. Certain studies have explored the association between molecular subtypes and sentinel lymph node metastasis and risk factors of metastasis (4-14). However, breast cancer is a highly heterogeneous disease at the molecular level and the metastatic behavior of different subtypes of breast cancer, including metastatic sites and the number of metastases, as well as the prognosis difference between primary and metastatic breast cancer patients of different subtypes, have remained to be fully defined.

In the present study, the possible association between molecular subtypes and metastatic behavior was retrospectively explored in a single-center sample of 166 patients with metastatic breast cancer from Hunan Provincial People's Hospital (Changsha, China) between January 2012 and December 2018, and the results were further supported by analysis of a large dataset from the Surveillance, Epidemiology, and End Results (SEER) database with 15,322 cases of metastatic breast cancer among 329,770 patients with primary breast cancer. 
Furthermore, differences in prognosis regarding overall survival(OS) of patients with primary and with metastatic breast cancer with different molecular subtypes were determined. The results of the present study may be valuable to inform improved monitoring for metastasis sites during follow-up. An understanding of the patterns of metastatic spread will allow the clinician to make more efficient surveillance decisions and select appropriate examinations and therapies.

\section{Materials and methods}

Patients. The present study was a retrospective analysis. First, 166 patients with metastatic breast cancer treated at Hunan Provincial People's Hospital (Changsha, China) between January 2012 and December 2018 were collected. All patients underwent primary tumor resection and the tumors were pathologically confirmed as breast cancer. The age at diagnosis was 25-79 years. Each patient included had a complete medical record and preserved pathological specimens. The study protocol was approved by the Ethics Committee of Hunan Provincial People's Hospital and The First Affiliated Hospital of Hunan Normal University (Changsha, China).

Furthermore, data were extracted from the SEER database (https://seer.cancer.gov/) on 407,791 patients who were diagnosed with malignant breast cancer between 2010 and 2016 and had known HR and human epidermal growth factor receptor 2 (HER2) statuses. After excluding non-primary breast cancer cases, there were 329,770 primary breast cancer patients, of which 15,322 cases had metastatic breast cancer. The number of HR+/HER2- metastatic cases was 9,222, compared with 2,710 HR+/HER2+ metastatic cases, 1,406 HR-/HER2+ metastatic cases and 1,984 HR-/HER2- metastatic cases. Bone metastasis was present in 11,299 cases, as compared with 5,207 cases with lung metastasis, 4,404 with liver metastasis, 1,201 with brain metastasis and 751 with distant lymph node (DLN) metastasis, as well as 453 cases that involved other metastatic sites (Fig. 1).

Characterization of specimens. Tumor specimen samples were processed by routine detection methods. First, tumor samples were fixed, after which they were consecutively sliced to a thickness of $4 \mu \mathrm{m}$ and then stained with H\&E, followed by immunohistochemistry (IHC) staining. Fluorescence in situ hybridization (FISH) was performed when necessary. After staining, the HR and HER2 statuses of all specimens were assessed by two senior pathologists based on the same criteria as those used by the hospital.

For evaluation, the following specific standards were used: A total of 5 high-power fields were randomly selected to count $>500$ cells and the number of positive cells and staining intensity were used for scoring. Samples were considered HR+ if $>1 \%$ of cells exhibited IHC staining (15). HER2 expression was only present on the cell membrane or in the cell plasma, and samples scored 3+ (overexpression) via IHC were classified as HER2+. For those samples scoring as HER2 1-2+, 2+ and 2-3+ on IHC, FISH was further performed by hybridization of fluorescent DNA probes to the HER2 gene and chromosome 17. The result was considered HER2+ status when the HER2/centromeric probe for chromosome 17 (Cep17) ratio was $\geq 2$ on average for 60 cells and detection of gene amplification was interpreted as HER2+ status (16).

Study design. The patients were divided into four groups according to molecular subtypes: HR+/HER2-, HR+/HER2+, HR-/HER2+ and HR-/HER2-. First, 166 patients with metastatic breast cancer with HR and HER2 expression data were assessed. The associations between molecular subtypes and the sites of and the number of metastases were examined.

Furthermore, 15,322 metastatic cases among 329,770 patients with primary breast cancer from the SEER database were analyzed. The possible impact of molecular subtypes on the sites and number of metastases was studied based on data from the SEER database. In addition, the possible impact of molecular subtypes on the OS of patients with primary and with metastatic breast cancer was evaluated. OS was defined as the time from the date of diagnosis to death from any cause.

Statistical analysis. All data were analyzed by using SPSS version 22.0 (IBM Corp.), where ratio variables were compared using the $\chi^{2}$ test or Fisher's exact test as appropriate. Survival rates were calculated with the Kaplan-Meier method and survival curves were drawn. The log-rank test was performed to perform univariate analysis. The statistical analyses were two-sided and $\mathrm{P}<0.05$ was considered to indicate statistical significance.

\section{Results}

Major characteristics of patients with different molecular subtypes of breast cancer. A total of 166 patients with metastatic breast cancer from the Hunan Provincial People's Hospital (Changsha, China) and 15,322 metastatic breast cancer cases from the SEER database were identified. The major baseline features of the breast cancer molecular subtypes are presented in Tables I and II. In 166 patients with metastases, the HR+/HER2-, HR+/HER2+, HR-/HER2+ and HR-/HER2- cases accounted for 55.4, 15.7, 12.6 and 16.3\%, respectively; all of the patients were female, with a mean age of 51.4 years (range, 25-79 years). For the 15,322 metastatic breast cancer cases from the SEER database, the mean age was 60.7 years (range, 19-103 years); the HR+/HER2-, HR+/HER2+, HR-/HER2+ and HR-/HER2cases accounted for $60.2,17.7,9.2$ and $12.9 \%$, respectively. In both datasets, HR+/HER2- accounted for the largest proportion of molecular subtypes, while HR-/HER2+ had the smallest proportion. There was no statistically significant difference in the proportion of molecular subtypes between the two datasets $(\mathrm{P}=0.196$; Table III).

Molecular subtypes and distribution of metastatic sites in the two datasets. Among the 166 cases of the present single-center study, the molecular subtypes were significantly associated with the prevalence of DLN metastasis $(\mathrm{P}=0.010)$, but not significantly associated with the prevalence of any other sites of metastasis. Bone metastasis was more common in the HR+/HER2+ and HR-/HER2+ subtypes compared to the other subtypes and their rates of occurrence were 50.0 and $42.9 \%$, respectively. Lung metastasis was more common in the HR+/HER2- and HR-/HER2+ subtypes, and their rates of occurrence were 23.9 and $23.8 \%$ respectively; furthermore, 


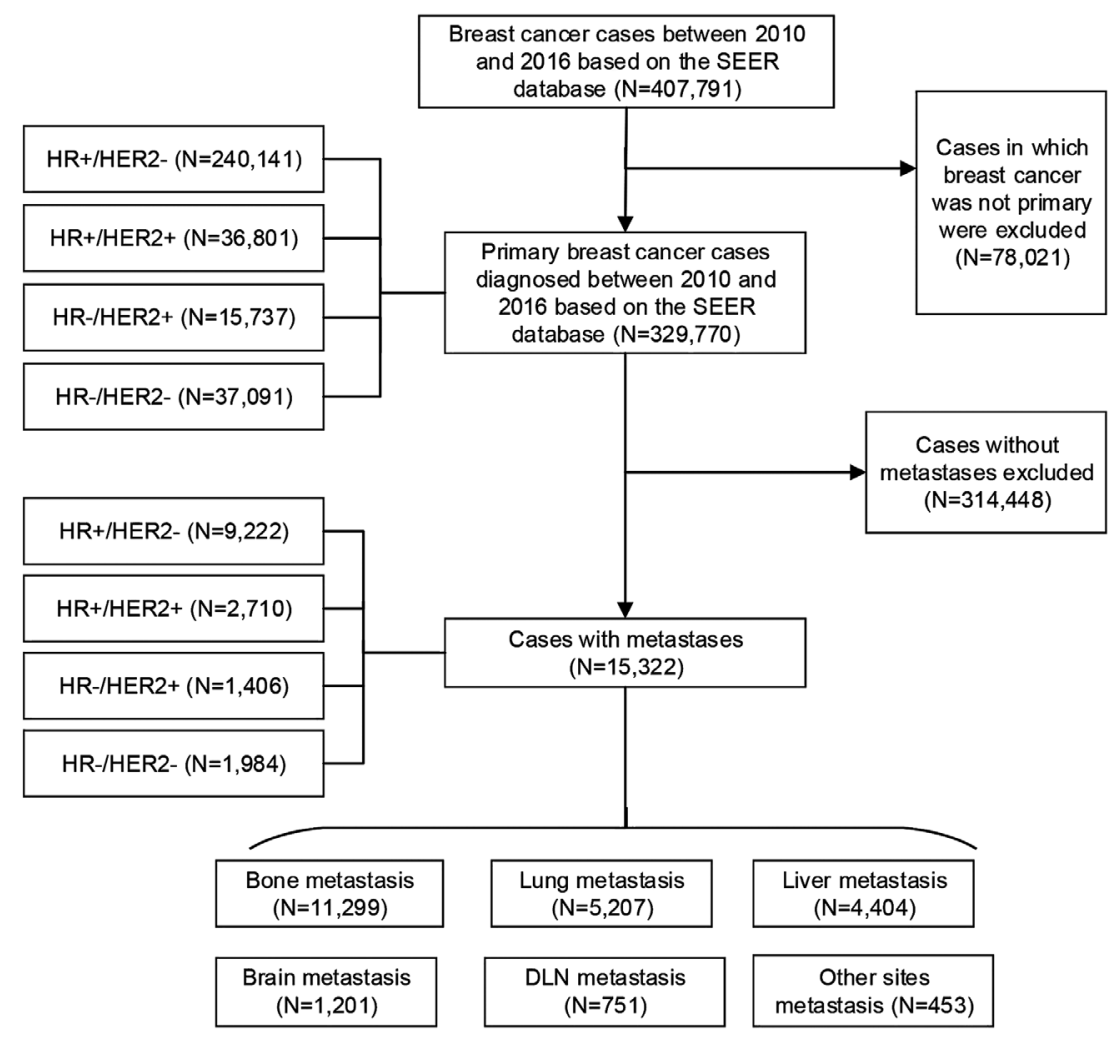

Figure 1. Flowchart of patient inclusion in the present cohort study based on the SEER database. SEER, Surveillance, Epidemiology and End Results; HR, hormone receptor; HER2, human epidermal growth factor receptor 2; DLN, distant lymph node.

pleural metastasis was more common in the HR+/HER2- and HR-/HER2-subtypes, and their rates of occurrence were 19.6 and $18.5 \%$, respectively. Liver metastasis was more common in the HR-/HER2+ and HR+/HER2+ subtypes and their rates of occurrence were 33.3 and $30.8 \%$, respectively. Brain metastasis was more common in the HR+/HER2+ and HR+/HER2- subtypes and their rates of occurrence were 11.5 and $5.4 \%$ respectively; DLN metastasis was also more common in the HR-/HER2- and HR-/HER2+ subtypes and their rates of occurrence were 44.4 and $23.8 \%$, respectively (Table IV).

Among the 15,322 metastatic cases from the SEER database, the metastasis sites were significantly different among the different molecular subtypes (bone, lung, liver, brain and DLN; $\mathrm{P}<0.001)$. Bone metastasis was more common in the HR+/HER2- and HR+/HER2+ than in the other subgroups, with rates of occurrence of 82.0 and $71.3 \%$, respectively. Lung metastasis was more common in the HR-/HER2+ and HR-/HER2- subtypes and the rates of occurrence were 40.8 and $47.7 \%$, respectively. Liver metastasis was more common in the HR-/HER2+ and HR+/HER2+ subtypes, with rates of occurrence of 51.6 and $39.2 \%$, respectively; brain metastasis was more common in the HR-/HER2+ and HR-/HER2- subtypes and the rates of occurrence were 12.7 and $12.9 \%$, respectively. DLN metastasis was more common in the HR-/HER2- and HR-/HER2+ subtypes, with rates of occurrence of 6.4 and $6.9 \%$, respectively (Table V).

In summary, bone metastasis was more likely to occur in the HR+/HER2+ subtype patients according to the single-center data as well as the dataset from the SEER database. Lung metastasis was more likely to occur in patients of the HR-/HER2+ subtype in both datasets. Liver metastasis was more likely to occur in the HR-/HER2+ and HR+/HER2+ patients in both datasets. DLN metastasis was more likely to occur in the HR-/HER2- and HR-/HER2+ patients in both datasets.

Molecular subtypes and the number of metastatic sites in both datasets. In the 166 cases from the single-center cohort, molecular subtypes were not significantly associated with the prevalence of the number of metastatic sites $(\mathrm{P}=0.221)$. A single site of metastasis was more frequent in the HR+/HER2and HR-/HER2+ patients than in the other subgroups, with frequencies of 59.8 and $57.1 \%$, respectively. Furthermore, two sites of metastasis were more common in the HR-/HER2- and HR+/HER2+ patients and the rates of occurrence were 37.0 and $30.8 \%$, respectively. In addition, three sites of metastasis were more frequent in HR+/HER2+ and HR+/HER2-patients, with rates of occurrence of 19.2 and $10.9 \%$, respectively. Finally, $\geq 4$ sites of metastasis were more frequent in the HR-/HER2- and HR-/HER2+ subtypes and the rates of occurrence were 14.8 and $14.3 \%$, respectively (Table VI).

Among the 15,322 metastatic cases from the SEER database, the molecular subtypes were significantly associated with the prevalence of the number of metastatic sites $(\mathrm{P}<0.001)$. A single site of metastasis was more common in the HR+/HER2- and HR-/HER2- patients than in the other subgroups, with rates of occurrence of 65.5 and $60.5 \%$, respectively. Furthermore, two sites of metastasis were more frequent in the HR+/HER $2+$ and HR-/HER $2+$ patients and the rates of occurrence were 28.6 and $28.4 \%$, respectively. In addition, three sites of metastasis were more common in the HR-/HER2+ and HR+/HER 2+ subtypes, with rates of occurrence of 13.0 and $11.5 \%$, respectively. Finally, $\geq 4$ sites 
Table I. Major characteristics of the patients with metastatic breast cancer from the present cohort study $(n=166)$.

\begin{tabular}{|c|c|c|c|c|}
\hline Main characteristics & HR+/HER2- $(n=92)$ & $\mathrm{HR}+/ \mathrm{HER} 2+(\mathrm{n}=26)$ & HR-/HER2+ $(n=21)$ & HR-/HER2- $(n=27)$ \\
\hline \multicolumn{5}{|l|}{ Age (years) } \\
\hline$<40$ & $19(20.6)$ & $5(19.2)$ & $2(9.5)$ & $1(3.7)$ \\
\hline $40-49$ & $26(28.3)$ & $8(30.8)$ & $3(14.3)$ & $9(33.3)$ \\
\hline $50-59$ & $26(28.3)$ & $6(23.1)$ & $9(42.9)$ & $11(40.8)$ \\
\hline$\geq 60$ & $21(22.8)$ & $7(26.9)$ & $7(33.3)$ & $6(22.2)$ \\
\hline \multicolumn{5}{|l|}{ Menopausal status } \\
\hline Premenopause & $41(44.6)$ & $12(46.2)$ & $5(23.8)$ & $9(33.3)$ \\
\hline Postmenopause & $51(55.4)$ & $14(53.8)$ & $16(76.2)$ & $18(66.7)$ \\
\hline \multicolumn{5}{|l|}{ Tumor stage } \\
\hline $\mathrm{T} 1$ & $9(9.8)$ & $5(19.2)$ & $4(19.1)$ & $8(29.6)$ \\
\hline $\mathrm{T} 2$ & $55(59.8)$ & $11(42.3)$ & $8(38.1)$ & $15(55.6)$ \\
\hline $\mathrm{T} 3$ & $18(19.6)$ & $9(34.6)$ & $7(33.3)$ & $3(11.1)$ \\
\hline $\mathrm{T} 4$ & $10(10.8)$ & $1(3.9)$ & $2(9.5)$ & $1(3.7)$ \\
\hline \multicolumn{5}{|l|}{ TNM stage } \\
\hline I & $7(7.6)$ & $4(15.4)$ & $0(0)$ & $3(11.1)$ \\
\hline II & $34(37.0)$ & $7(26.9)$ & $6(28.6)$ & $12(44.4)$ \\
\hline III & $51(55.4)$ & $15(57.7)$ & $15(71.4)$ & $12(44.4)$ \\
\hline \multicolumn{5}{|l|}{ Lymph node status } \\
\hline Positive & $65(70.7)$ & $19(73.1)$ & $19(90.5)$ & $16(59.3)$ \\
\hline Negative & $27(29.3)$ & $7(26.9)$ & $2(9.5)$ & $11(40.7)$ \\
\hline \multicolumn{5}{|l|}{ Histopathological type } \\
\hline Invasive ductal carcinoma & $75(81.5)$ & $23(88.5)$ & $18(85.7)$ & $25(92.6)$ \\
\hline Invasive lobular carcinoma & $4(4.3)$ & $0(0)$ & $0(0)$ & $1(3.7)$ \\
\hline Ductal carcinoma in situ & $2(2.2)$ & $2(7.7)$ & $1(4.8)$ & $0(0)$ \\
\hline Other & $11(12.0)$ & $1(3.8)$ & $2(9.5)$ & $1(3.7)$ \\
\hline
\end{tabular}

Values are expressed as n (\%). HR, hormone receptor; HER2, human epidermal growth factor receptor 2.

of metastasis were more common for the HR-/HER2+ and HR-/HER2-subtypes (4.5 and 3.5\%, respectively; Table VII).

The results of both datasets all suggested that the number of metastatic sites was diverse across the molecular subtypes. A single site of metastasis was more likely in HR+/HER2-patients, while 2-3 sites of metastases were more likely in $\mathrm{HR}+/ \mathrm{HER} 2+$ patients. Furthermore, $\geq 4$ sites of metastasis were more likely in patients with the HR-/HER2+ and HR-/HER2- subtype.

Subsequently, the information on all combinations of different distant metastases was further analyzed in the subtypes of patients in both datasets. In both datasets, it was indicated that regarding single distant metastasis, bone-only metastasis was more common in the HR+/HER2-subtype $(\mathrm{P}<0.05)$ and liver-only metastasis was more common in the HR+/HER2+ and HR-/HER $2+$ subtypes $(\mathrm{P}<0.05)$, while DLN-only metastasis was more common in HR-/HER2- patients $(\mathrm{P}<0.05)$. Regarding the combinations of distant metastases to two different sites, bone + liver metastases were more common in the HR+/HER 2+ subtype and bone + brain metastases were also more common in the HR+/HER2+ subtype, while lung + brain metastases were more common in the HR-/HER2- subtype. For combinations of metastasis to three different distant sites, bone + lung + DLN metastases were more common in the HR+/HER2- subtype (Figs. 2 and 3; Tables SI and SII).

Molecular subtypes and the OS of patients with primary and metastatic breast cancer based on the SEER database. Based on the results discussed in the previous section, the influence of the molecular subtype on the OS of 329,770 primary breast cancer patients from the SEER database between 2010 and 2016 was first analyzed. There was a statistical difference in OS among the molecular subtypes with primary breast cancer $(\mathrm{P}<0.001)$. The mean OS period of $\mathrm{HR}+/ \mathrm{HER} 2-$ patients was 78.5 months and the mean OS period of HR+/HER2+, HR-/HER2+ and HR-/HER2- patients was 76.6, 72.9 and 69.1 months, respectively (Fig. 4A).

Furthermore, the association between molecular subtypes of the 15,322 metastatic breast cancer patients from the SEER database between 2010 and 2016 and OS was determined. There was a statistically significant difference in OS among the different molecular subtypes with metastasis $(\mathrm{P}<0.001)$. The mean OS of patients with metastasis of the HR+/HER2-type was 40.2 months, while that of the HR+/HER2+, HR-/HER2+ and HR-/HER2-types was 46.0, 40.4 and 18.5 months, respectively (Fig. 4B). 
Table II. Major characteristics of patients with metastatic breast cancer from the Surveillance, Epidemiology and End Results dataset $(\mathrm{n}=15,322)$.

\begin{tabular}{|c|c|c|c|c|}
\hline Main characteristics & $\begin{array}{c}\text { HR+/HER2- } \\
\quad(n=9,222)\end{array}$ & $\begin{array}{c}\mathrm{HR}+/ \mathrm{HER} 2+ \\
\quad(\mathrm{n}=2,710)\end{array}$ & $\begin{array}{l}\text { HR-/HER2+ } \\
\quad(n=1,406)\end{array}$ & $\begin{array}{l}\text { HR-/HER2- } \\
(\mathrm{n}=1,984)\end{array}$ \\
\hline \multicolumn{5}{|l|}{ Age (years) } \\
\hline$<40$ & $553(6.0)$ & $298(11.0)$ & $169(12.0)$ & $160(8.1)$ \\
\hline $40-49$ & $1,193(12.9)$ & $430(15.9)$ & $227(16.2)$ & $289(14.6)$ \\
\hline $50-59$ & $2,178(23.6)$ & 765 (28.2) & $411(29.2)$ & $512(25.8)$ \\
\hline$\geq 60$ & $5298(57.5)$ & $1,217(44.9)$ & $599(42.6)$ & $1,023(51.5)$ \\
\hline \multicolumn{5}{|l|}{ Sex } \\
\hline Female & $9,107(98.8)$ & $2,673(98.6)$ & $1,403(99.8)$ & $1969(99.2)$ \\
\hline Male & $115(1.2)$ & $37(1.4)$ & $3(0.2)$ & $15(0.8)$ \\
\hline \multicolumn{5}{|l|}{ Tumor stage } \\
\hline $\mathrm{T} 0$ & $110(1.2)$ & $23(0.8)$ & $15(1.1)$ & $26(1.3)$ \\
\hline $\mathrm{T} 1$ & $819(8.9)$ & $224(8.3)$ & $99(7.0)$ & $133(6.7)$ \\
\hline $\mathrm{T} 2$ & $2,287(24.8)$ & $668(24.6)$ & $278(19.8)$ & $416(21.0)$ \\
\hline $\mathrm{T} 3$ & $1,188(12.9)$ & $345(12.7)$ & $190(13.5)$ & $301(15.2)$ \\
\hline $\mathrm{T} 4$ & $2,244(24.3)$ & $713(26.3)$ & 449 (31.9) & $617(31.1)$ \\
\hline Unknown & $2,574(27.9)$ & $737(27.3)$ & $375(26.7)$ & $491(24.7)$ \\
\hline \multicolumn{5}{|l|}{ Lymph node status } \\
\hline Positive & $8,919(96.7)$ & $2,563(94.6)$ & $1,296(92.2)$ & $1,819(91.7)$ \\
\hline Negative & $303(3.3)$ & $147(5.4)$ & $110(7.8)$ & $165(8.3)$ \\
\hline \multicolumn{5}{|l|}{ Grade } \\
\hline I & $894(9.7)$ & $62(2.3)$ & $7(0.5)$ & $23(1.2)$ \\
\hline II & $3,855(41.8)$ & $891(32.9)$ & $310(22.0)$ & $296(14.9)$ \\
\hline III & $2,604(28.2)$ & $1,326(48.9)$ & $839(59.7)$ & $1,333(67.2)$ \\
\hline IV & $34(0.4)$ & $16(0.6)$ & $11(0.8)$ & $25(1.3)$ \\
\hline Unknown & $1,835(19.9)$ & $415(15.3)$ & $239(17.0)$ & $307(15.4)$ \\
\hline \multicolumn{5}{|l|}{ Radiation therapy } \\
\hline Prior to surgery & $146(1.6)$ & $37(1.4)$ & $20(1.4)$ & $22(1.1)$ \\
\hline After surgery & $1,343(14.6)$ & $409(15.1)$ & $209(14.9)$ & $315(15.9)$ \\
\hline Prior to and after surgery & $39(0.4)$ & $8(0.3)$ & $7(0.5)$ & $7(0.4)$ \\
\hline None & $7,678(83.3)$ & $2,250(83.0)$ & $1,166(82.9)$ & $1,636(82.4)$ \\
\hline Other & $16(0.1)$ & $6(0.2)$ & $4(0.3)$ & $4(0.2)$ \\
\hline \multicolumn{5}{|l|}{ Chemotherapy } \\
\hline No & $5,058(54.8)$ & 748 (27.6) & $288(20.5)$ & $553(27.9)$ \\
\hline Yes & $4,164(45.2)$ & $1,962(72.4)$ & $1,118(79.5)$ & $1,431(72.1)$ \\
\hline
\end{tabular}

Values are expressed as n (\%). HR, hormone receptor; HER2, human epidermal growth factor receptor 2.

\section{Discussion}

In most cases, the generation of breast cancer metastatic lesions may last for months, years or even decades prior to becoming a clinically detectable metastasis (17). While the underlying mechanisms remain to be fully elucidated, it is known that metastasis is a process that begins with the detachment of tumor cells from the primary tumor (18). According to the St. Gallen International Expert Consensus on the Primary Therapy of Early Breast Cancer in 2011 (19), given the limitation of molecular profiling studies in routine clinical practice, the combination of the HR and HER 2 status with or without an accompanying Ki-67 proliferation index has been recently used as an indicator for molecular subtypes. Different molecular subtypes have distinctive biological features and clinical outcomes (20). Molecular subtypes remain the most important prognostic determinants in breast cancer (21). The goal of the present study was to explore the possible impact of molecular subtypes on metastatic behavior and OS in a single-center study combined with a large cohort study from the SEER database. Monitoring the biological behavior of breast cancer may benefit a patient by allowing for the implementation of a personalized treatment strategy (22). 
Table III. Similarities and differences of molecular subtypes between the two datasets.

\begin{tabular}{lcccr}
\hline & & $\begin{array}{c}\text { Surveillance, Epidemiology } \\
\text { and End Results } \\
\text { dataset }(\mathrm{n}=15,322)\end{array}$ & $\chi^{2}$ & P-value \\
\hline HR+/HER2- & Single-centerdataset $(\mathrm{n}=166)$ & $9,222(60.2)$ & 0.196 \\
HR+/HER2+ & $92(55.4)$ & $2,710(17.7)$ & \\
HR-/HER2+ & $26(15.7)$ & $1,406(9.2)$ & \\
HR-/HER2- & $21(12.6)$ & $1,984(12.9)$ & \\
\hline
\end{tabular}

Values are expressed as $\mathrm{n}(\%)$. HR, hormone receptor; HER2, human epidermal growth factor receptor 2. P-value pertains to comparison among all groups.

Table IV. Molecular subtypes and metastatic sites in the patients with metastatic breast cancer from the present cohort study $(n=166)$.

\begin{tabular}{|c|c|c|c|c|c|}
\hline Metastatic site & HR+/HER2- $(n=92)$ & $\mathrm{HR}+/ \mathrm{HER} 2+(\mathrm{n}=26)$ & HR-/HER2+ $(n=21)$ & HR-/HER2- (n=27) & P-value \\
\hline Bone & & & & & 0.165 \\
\hline Metastasis & $31(33.7)$ & $13(50.0)$ & $9(42.9)$ & $6(22.2)$ & \\
\hline No metastasis & $61(66.3)$ & $13(50.0)$ & $12(57.1)$ & $21(77.8)$ & \\
\hline Lung & & & & & 0.779 \\
\hline Metastasis & $22(23.9)$ & $4(15.4)$ & $5(23.8)$ & $5(18.5)$ & \\
\hline No metastasis & $70(76.1)$ & $22(84.6)$ & $16(76.2)$ & $22(81.5)$ & \\
\hline Pleural & & & & & 0.330 \\
\hline Metastasis & 15 (19.6) & $1(3.8)$ & $2(9.5)$ & $5(18.5)$ & \\
\hline No metastasis & $77(80.4)$ & $25(96.2)$ & $19(90.5)$ & $22(81.5)$ & \\
\hline Liver & & & & & 0.438 \\
\hline Metastasis & $18(19.6)$ & $8(30.8)$ & $7(33.3)$ & $6(22.2)$ & \\
\hline No metastasis & $74(80.4)$ & $18(69.2)$ & $14(66.7)$ & $21(77.8)$ & \\
\hline Brain & & & & & 0.425 \\
\hline Metastasis & $5(5.4)$ & $3(11.5)$ & $0(0.0)$ & $1(3.7)$ & \\
\hline No metastasis & 87 (94.6) & $23(88.5)$ & $21(100.0)$ & $26(96.3)$ & \\
\hline DLN & & & & & 0.010 \\
\hline Metastasis & 18 (19.6) & $2(7.7)$ & $5(23.8)$ & $12(44.4)$ & \\
\hline No metastasis & $74(80.4)$ & $24(92.3)$ & $16(76.2)$ & $15(55.6)$ & \\
\hline
\end{tabular}

Values are expressed as $\mathrm{n}(\%)$. Pleural refers to the pleural space or sac. HR, hormone receptor; HER2, human epidermal growth factor receptor 2; DLN, distant lymph node. P-value pertains to comparison among all groups.

The present study indicated that bone metastasis is more frequent in HR+/HER2+ patients, while metastasis to the lung is more frequent in the HR-/HER2+ subtype as compared to the other molecular subtypes of patients with breast cancer. Smid et al (6) also determined that bone metastasis is most abundant in the luminal subtypes. Largely in accordance with these observations, tissue microarray analysis suggested that the HER2 subtype exhibited higher rates of lung metastasis compared with luminal A cancers (7). The luminal B subtype was less frequently associated with lung metastasis than the HER2 subtype (23). The liver was a common organ involved in breast cancer metastasis. A previous study reported that liver metastasis was more frequently observed in the HER2 subtype than the luminal A and triple-negative breast cancer (TNBC) subtypes (23). Similarly, the present study indicated that liver metastasis was more likely to be present in the HR-/HER2+ and HR+/HER2+ subtypes from both datasets. However, only a small number of previous studies have investigated pleural metastases. One previous study reported that the luminal A and B subtypes were both less frequently associated with pleural metastasis than the TNBC subtype (23). In the present study, the HR+/HER2- and HR-/HER2- subtypes had an increased likelihood to have pleural metastasis in the single-center cohort. Since the metastatic sites recorded in the SEER database did not include the pleura, it was not possible to use those big data to further support this result. 
Table V. Molecular subtypes and metastatic sites in patients with metastatic breast cancer from the Surveillance, Epidemiology and End Results dataset $(\mathrm{n}=15,322)$.

\begin{tabular}{|c|c|c|c|c|c|}
\hline Metastatic sites & HR+/HER2- $(n=9,222)$ & $\mathrm{HR}+/ \mathrm{HER} 2+(\mathrm{n}=2,710)$ & HR-/HER2+ $(n=1,406)$ & HR-/HER2- $(\mathrm{n}=1,984)$ & P-value \\
\hline Bone & & & & & $<0.001$ \\
\hline Metastasis & $7,563(82.0)$ & $1,931(71.3)$ & $762(54.2)$ & $1,043(52.6)$ & \\
\hline No metastasis & $1,659(18.0)$ & 779 (28.7) & $644(45.8)$ & $941(47.4)$ & \\
\hline Lung & & & & & $<0.001$ \\
\hline Metastasis & $2,787(30.2)$ & $901(33.2)$ & $573(40.8)$ & 946 (47.7) & \\
\hline No metastasis & $6,435(69.8)$ & $1,809(66.8)$ & $833(59.2)$ & $1,038(52.3)$ & \\
\hline Liver & & & & & $<0.001$ \\
\hline Metastasis & $1,965(21.3)$ & $1,062(39.2)$ & $726(51.6)$ & $651(32.8)$ & \\
\hline No metastasis & $7,257(78.7)$ & $1,648(60.8)$ & $680(48.4)$ & $1,333(67.2)$ & \\
\hline Brain & & & & & $<0.001$ \\
\hline Metastasis & $542(5.9)$ & $226(8.3)$ & $178(12.7)$ & $255(12.9)$ & \\
\hline No metastasis & $8,680(94.1)$ & $2,484(91.7)$ & $1,228(87.3)$ & $1,729(87.1)$ & \\
\hline DLN & & & & & $<0.001$ \\
\hline Metastasis & $363(3.9)$ & $165(6.1)$ & $97(6.9)$ & $126(6.4)$ & \\
\hline No metastasis & $8,859(96.1)$ & $2,545(93.9)$ & $1,309(93.1)$ & $1,858(93.6)$ & \\
\hline
\end{tabular}

Values are expressed as n (\%). HR, hormone receptor; HER2, human epidermal growth factor receptor 2; DLN, distant lymph node. P-value pertains to comparison among all groups.

Table VI. Molecular subtypes and the number of metastatic sites in patients with metastatic breast cancer from the present cohort study $(\mathrm{n}=166)$.

\begin{tabular}{lccccc}
\hline $\begin{array}{l}\text { Number of } \\
\text { metastatic sites }\end{array}$ & $\begin{array}{c}\text { HR+/HER2- } \\
(\mathrm{n}=92)\end{array}$ & $\begin{array}{c}\text { HR+/HER2+ } \\
(\mathrm{n}=26)\end{array}$ & $\begin{array}{c}\text { HR-/HER2+ } \\
(\mathrm{n}=21)\end{array}$ & $\begin{array}{c}\text { HR-/HER2- } \\
(\mathrm{n}=27)\end{array}$ & $\chi^{2}$ \\
\hline 1 & $55(59.8)$ & $12(46.2)$ & $12(57.1)$ & $13(48.2)$ & 11.328 \\
2 & $20(21.7)$ & $8(30.8)$ & $4(19.1)$ & $10(37.0)$ & 0.221 \\
3 & $10(10.9)$ & $5(19.2)$ & $2(9.5)$ & $0(0)$ \\
$\geq 4$ & $7(7.6)$ & $1(3.8)$ & $3(14.3)$ & $4(14.8)$ \\
\hline
\end{tabular}

Values are expressed as n (\%). HR, hormone receptor; HER2, human epidermal growth factor receptor 2. P-value pertains to comparison among all groups.

The brain metastasis of breast cancer is not common (6); the present study indicated that metastasis to the brain was less common than metastasis to other organs. In the present study, the HR-/HER2+ and HR-/HER2-subtypes were observed to have relatively more brain metastasis than the other two subtypes. This result is consistent with a previous study, which indicated that the HR-/HER2+ subtype had a higher risk of brain metastasis (5).

There may be various reasons why different molecular subtypes of breast cancer exhibit differences in metastatic sites. A previous study reported that downregulation of E-cadherin was crucial to the dissemination and invasion of cancer cells, which may augment breast cancer metastasis to the bone (24). Zhang et al (25) recently identified differentially expressed DEGs and signaling pathways that may make a contribution towards the understanding of the pathological mechanisms of bone metastasis from breast cancer. For example, integrin binding sialoprotein, matrix metallopeptidase, TNF $\alpha$-induced protein 6 , dehydrogenase/reductase 3 , receptor interacting serine/threonine kinase 4, and CD200 had a diagnostic value for patients with breast cancer bone metastasis. There was evidence that the ability of breast cancer cells to activate osteoclasts is similar to that of normal glandular tissue of mammary epithelial cells during lactation; therefore, breast cancer cells have intrinsic properties that allow them to metastasize to bone tissue (26). Sphingosine kinase 1 (SPHK1) promoted lung metastasis by transcriptionally upregulating the expression of the metastasis-promoting gene fascin actin-building protein 1 via $\mathrm{NF}-\kappa \mathrm{B}$ activation, and targeting SPHK1 and NF- $\kappa \mathrm{B}$ using clinically-applicable inhibitors significantly inhibited aggressive mammary tumor growth and spontaneous lung metastasis in orthotopic syngeneic HR-/HER2- subtype mouse models (27). 
Table VII. Molecular subtypes and the number of metastatic sites in patients with metastatic breast cancer from the Surveillance, Epidemiology and End Results dataset $(n=15,322)$.

\begin{tabular}{lccccrr}
\hline $\begin{array}{l}\text { Number of } \\
\text { metastatic sites }\end{array}$ & $\begin{array}{c}\text { HR+/HER2- } \\
(\mathrm{n}=9,222)\end{array}$ & $\begin{array}{c}\text { HR+/HER2+ } \\
(\mathrm{n}=2,710)\end{array}$ & $\begin{array}{c}\text { HR-/HER2+ } \\
(\mathrm{n}=1,406)\end{array}$ & $\begin{array}{c}\text { HR-/HER2- } \\
(\mathrm{n}=1,984)\end{array}$ & $\chi^{2}$ & P-value \\
\hline 1 & $6,042(65.5)$ & $1,546(57)$ & $761(54.1)$ & $1,200(60.5)$ & 160.329 & $<0.001$ \\
2 & $2,289(24.8)$ & $774(28.6)$ & $399(28.4)$ & $541(27.3)$ & & \\
3 & $722(7.8)$ & $312(11.5)$ & $183(13.0)$ & $173(8.7)$ & $70(3.5)$ & \\
$\geq 4$ & $169(1.8)$ & $78(2.9)$ & $63(4.5)$ & $70)$ & \\
\hline
\end{tabular}

Values are expressed as $\mathrm{n}(\%)$. HR, hormone receptor; HER2, human epidermal growth factor receptor 2. P-value pertains to comparison among all groups.
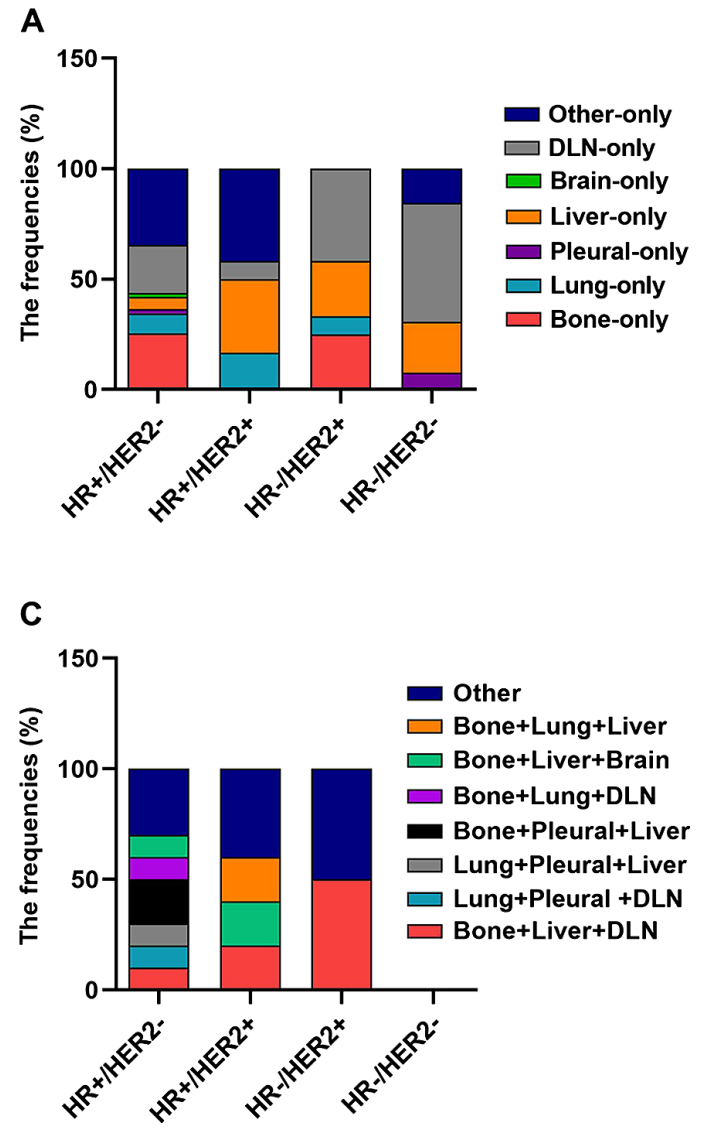

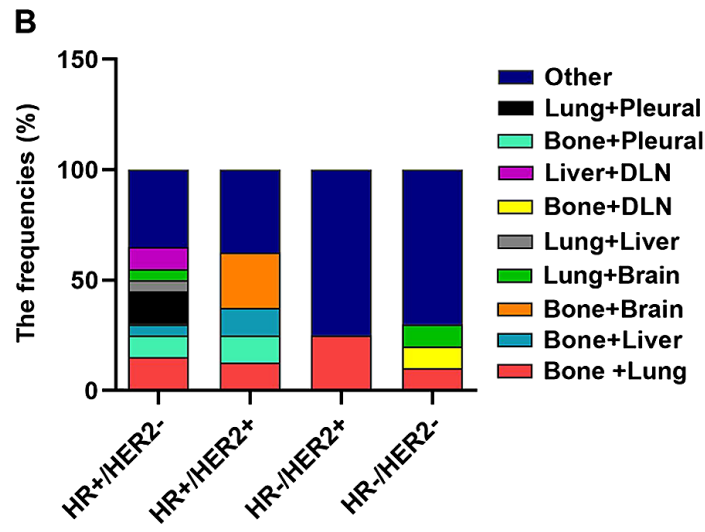

D

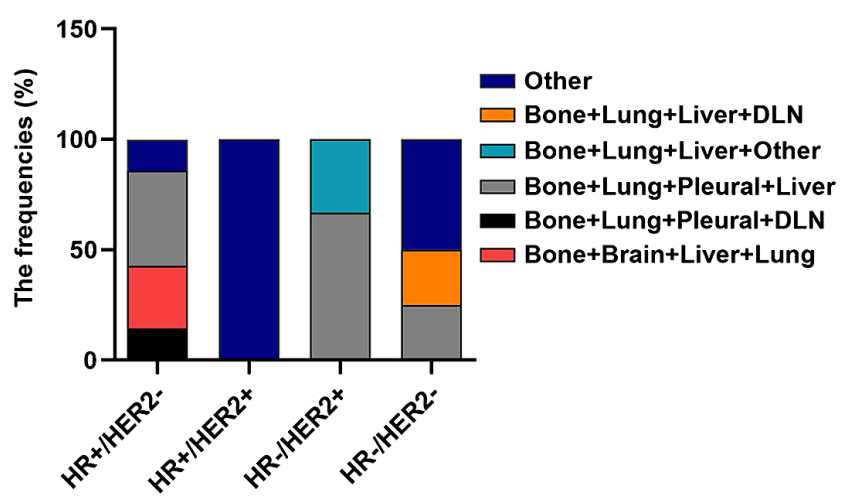

Figure 2. Frequencies of metastasis locations in 166 patients with metastatic breast cancer of the present cohort study according to molecular subtype. Frequencies of different metastatic sites in patients with breast cancer with involvement of (A) one, (B) two, (C) three and (D) four metastatic sites by each breast cancer subtype. DLN, distant lymph node; HR, hormone receptor; HER2, human epidermal growth factor receptor 2.

The mechanism for the tendency of HER2+ tumors to appear in the liver remains elusive. C-X-C motif chemokine receptor 4, a chemokine receptor enhanced by HER2 activation, has been proposed to be involved in promoting invasion of these cells to visceral organs (28). In addition, a previous study indicated that hepatic steatosis may serve as an independent factor to decrease liver metastasis in patients with breast cancer (29). The biological mechanisms of brain metastasis are currently unclear in breast cancer. A study reported that the WNT pathway was associated with relapse of breast cancer or metastasis to the brain (6). Future studies should investigate the metastatic mechanism in breast cancer across the different subtypes and develop strategies of how to reduce the overall risk of metastasis.

To date, the association of molecular subtypes with the number of metastatic sites in patients with breast cancer has been rarely investigated. In the present study, analysis of the single-center cohort and the SEER dataset both indicated that the the number of metastatic sites of the HR+/HER2- subtype patients was lower than in patients with the other three subtypes after those patients had experienced metastases, while the number of metastatic sites of the HR-/HER2+ and HR-/HER2- subtypes patients was higher. The present study 
A

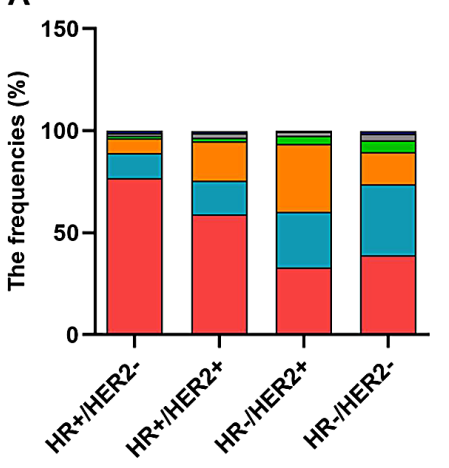

C

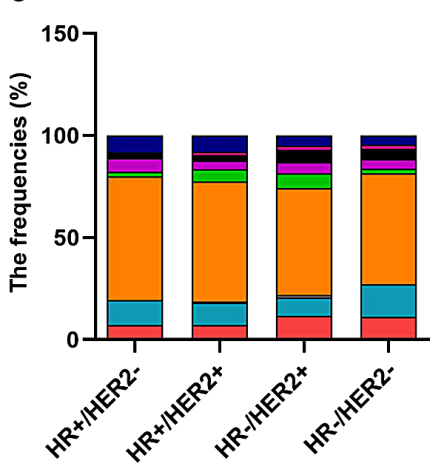

B

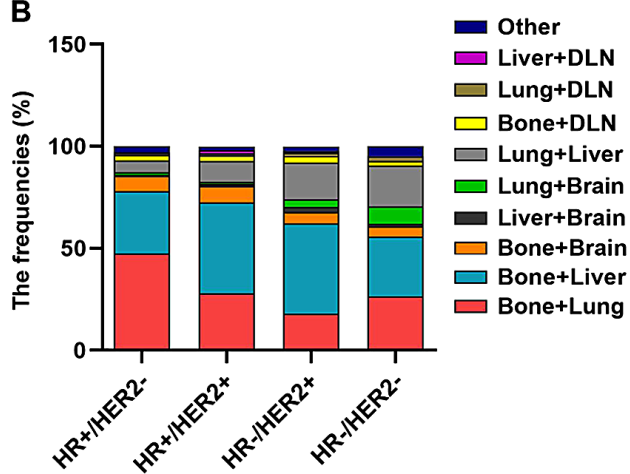

D

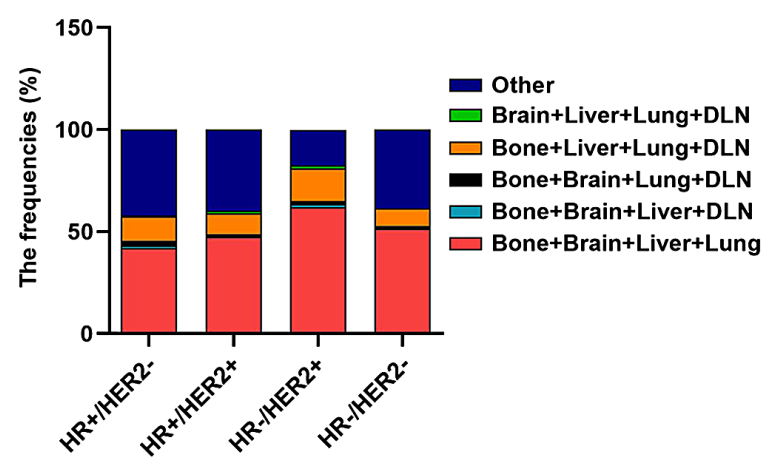

Figure 3. Frequencies of metastasis locations in patients with metastatic breast cancer from the Surveillance, Epidemiology and End Results dataset ( $\mathrm{n}=15,322)$ according to molecular subtype. Frequencies of different metastatic sites in patients with breast cancer with involvement of (A) one, (B) two, (C) three and (D) four metastatic sites by each breast cancer subtype. DLN, distant lymph node; HR, hormone receptor; HER2, human epidermal growth factor receptor 2.
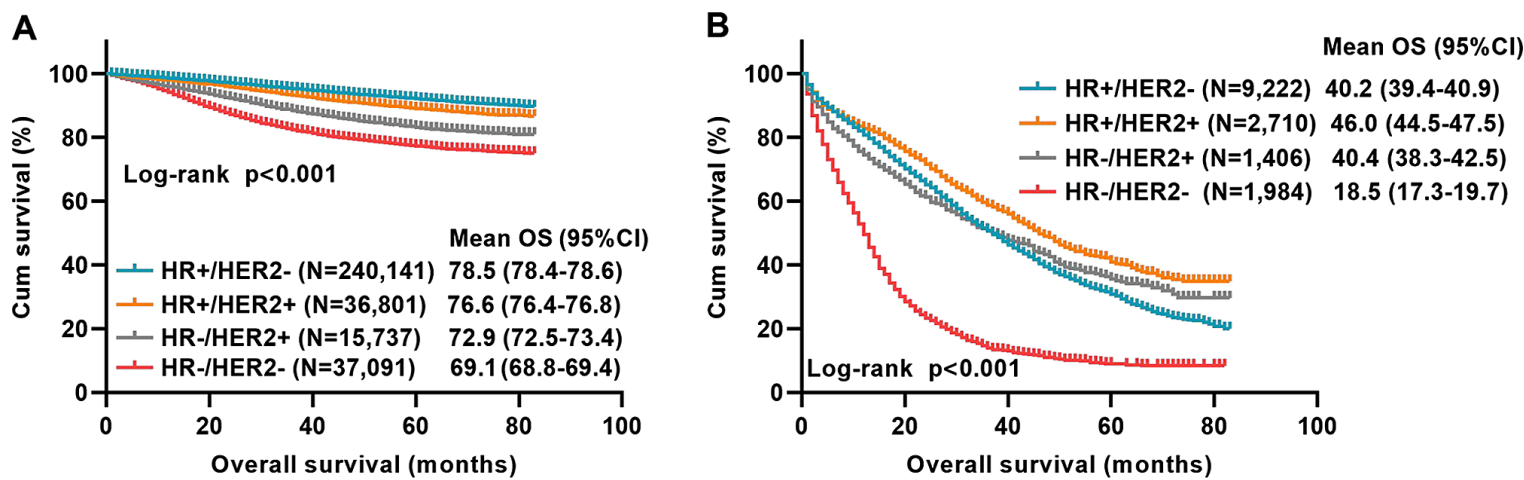

Figure 4. Influence of molecular subtypes on the OS of patients with primary breast cancer and with metastatic breast cancer based on the SEER database. (A) OS of 329,770 patients with primary breast cancer by molecular subtype; Log-rank P<0.001. (B) OS of 15,322 patients with metastatic breast cancer by molecular subtype; Log-rank P<0.001. OS, overall survival; SEER, Surveillance, Epidemiology and End Results; HR, hormone receptor; HER2, human epidermal growth factor receptor 2; Cum, cumulative survival. The definition of any censored datapoints: alive that their total time until death could not be determined; or dead of other cause; or missing.

lacks mechanistic evidence to explain why the molecular subtype affected the number of metastatic sites. Future work by our group will continue to explore the reasons for this and attempt to identify a possible mechanism.

Numerous studies have demonstrated different survival rates between molecular subtypes (30-32). However, the difference in prognosis between primary and metastatic tumors of different molecular subtypes remains to be fully elucidated. In the present study, there was a statistically significant difference in OS among the different molecular subtypes of patients with distant metastatic primary breast cancer between
2010 and 2016 from the SEER database. The patients of the HR+/HER2- subtype had the longest mean OS compared with the other three subtypes. HR-/HER2- was associated with a significantly poorer OS, whether in primary breast cancer or the subset of metastatic breast cancer patients. The Notch signaling pathway has emerged as a regulatory factor in the pathogenesis and tumor progression of TNBC (33). A previous study also reported that in a survival analysis of females diagnosed with de novo metastasis, the mortality risk relative to the HR+/HER2- subtype was twice as high for HR-/HER2and slightly lower for HR+/HER2+; HER2+ metastatic breast 
cancers had relatively better survival in recent years (34). The present study also determined that the mean OS of metastatic patients of the HR+/HER2+ subtype was the longest (46.0 months). Previous studies have indicated that the median survival time of patients with metastatic breast cancer was $\sim 2-4$ years (3) and the 5-year survival rate was only $25 \%$ (35). This was consistent with the present study.

As a limitation of the present study, no Kaplan-Meier analysis was performed for the present single-center cohort. As a proportion of metastatic breast cancer patients enrolled in the present single-center study between 2012 and 2016 were initially treated at Hunan Provincial People's Hospital (Changsha, China), when they were diagnosed with metastases later here, then they went back to their local hospitals for final treatment and some of them were lost to follow-up. In addition, some metastatic breast cancer patients enrolled in the present single-center study between 2017 and 2018 were still alive at the end of the follow-up that their total time until death could not be determined. Thus, considering the large amount of censored data in the present dataset, no corresponding prognostic survival analysis of the cases collected at Hunan Provincial People's Hospital (Changsha, China) was performed.

The present results may assist clinicians in the treatment of patients to select appropriate and standardized treatments. Although the results for the single-center cohort were not always consistent with the results obtained with the SEER data due to limited samples in the single-center cohort, the present results may be valuable for developing appropriate follow-up strategies and to guide personalized care.

In conclusion, the present study indicated that different molecular subtypes of breast cancer have different metastatic behaviors. The subtypes exhibited differences regarding the sites and number of metastases. The survival was different among the different molecular subtypes with metastasis. These results may assist clinicians in the prediction of metastatic behavior of breast cancers and develop more efficient follow-up monitoring strategies to further improve OS.

\section{Acknowledgements}

Not applicable.

\section{Funding}

This study was supported by The National Natural Science Foundation of China (grant no. 81572966).

\section{Availability of data and materials}

The datasets used and/or analyzed during the current study are available from the corresponding author on reasonable request.

\section{Authors' contributions}

Study design and supervision, as well as data curation were performed by WW. The manuscript was drafted by HY. Pathological work was performed by JZ and WG. Data collection and processing were performed by HY, RW, FRZ, SLP, YYM, SYC, SJD and LHZ. All authors read and approved the final manuscript.

\section{Ethics approval and consent to participate}

All procedures performed in this study were in accordance with the ethical standards of the institutional and national research committee and with the 1964 Helsinki declaration and its later amendments or comparable ethical standards. It was approved by the Medical Ethical Committee of Hunan Provincial People's Hospital and The First Affiliated Hospital of Hunan Normal University (Changsha, China) and written informed consent was provided by all of the participating patients.

\section{Patient consent for publication}

Not applicable.

\section{Competing interests}

The authors declare that they have no competing interests.

\section{References}

1. Bray F, Ferlay J, Soerjomataram I, Siegel RL, Torre LA and Jemal A: Global cancer statistics 2018: GLOBOCAN estimates of incidence and mortality worldwide for 36 cancers in 185 countries. CA Cancer J Clin 68: 394-424, 2018.

2. Santa-Maria CA, Nye L, Mutonga MB, Jain S and Gradishar WJ: Management of Metastatic HER2-positive breast cancer: Where are we and where do we go from here? Oncology (Williston Park) 30: 148-155, 2016

3. Puglisi F, Rea D, Kroes MA and Pronzato P: Second-line single-agent chemotherapy in human epidermal growth factor receptor 2-negative metastatic breast cancer: A systematic review. Cancer Treat Rev 43: 36-49, 2016.

4. Gerratana L, Fanotto V, Bonotto M, Bolzonello S, Minisini AM, Fasola G and Puglisi F: Pattern of metastasis and outcome in patients with breast cancer. Clin Exp Metastasis 32: 125-133, 2015.

5. Sihto H, Lundin J, Lundin M, Lehtimäki T, Ristimäki A, Holli K, Sailas L, Kataja V, Turpeenniemi-Hujanen T, Isola J, et al: Breast cancer biological subtypes and protein expression predict for the preferential distant metastasis sites: A nationwide cohort study. Breast Cancer Res 13: R87, 2011.

6. Smid M, Wang Y, Zhang Y, Sieuwerts AM, Yu J, Klijn JG, Foekens JA and Martens JW: Subtypes of breast cancer show preferential site of relapse. Cancer Res 68: 3108-3114, 2008.

7. Kennecke H, Yerushalmi R, Woods R, Cheang MC, Voduc D, Speers CH, Nielsen TO and Gelmon K: Metastatic behavior of breast cancer subtypes. J Clin Oncol 28: 3271-3277, 2010.

8. Berman AT, Thukral AD, Hwang WT, Solin LJ and Vapiwala N: Incidence and patterns of distant metastases for patients with early-stage breast cancer after breast conservation treatment. Clin Breast Cancer 13: 88-94, 2013.

9. Heitz F, Harter P, Lueck HJ, Fissler-Eckhoff A, Lorenz-Salehi F, Scheil-Bertram S, Traut A and du Bois A: Triple-negative and HER2-overexpressing breast cancers exhibit an elevated risk and an earlier occurrence of cerebral metastases. Eur J Cancer 45: 2792-2798, 2009.

10. Metzger-Filho O, Sun Z, Viale G, Price KN, Crivellari D, Snyder RD, Gelber RD, Castiglione-Gertsch M, Coates AS, Goldhirsch A and Cardoso F: Patterns of Recurrence and outcome according to breast cancer subtypes in lymph node-negative disease: Results from international breast cancer study group trials VIII and IX. J Clin Oncol 31: 3083-3090, 2013.

11. Paluch-Shimon S, Ben-Baruch N, Wolf I, Zach L, Kopolovic J, Kruglikova A, Modiano T, Yosepovich A, Catane R and Kaufman B: Hormone receptor expression is associated with a unique pattern of metastatic spread and increased survival among HER2-overexpressing breast cancer patients. Am J Clin Oncol 32: 504-508, 2009.

12. Dent R, Hanna WM, Trudeau M, Rawlinson E, Sun P and Narod SA: Pattern of metastatic spread in triple-negative breast cancer. Breast Cancer Res Treat 115: 423-428, 2009. 
13. Minisini AM, Moroso S, Gerratana L, Giangreco M, Iacono D, Poletto E, Guardascione M, Fontanella C, Fasola G and Puglisi F: Risk factors and survival outcomes in patients with brain metastases from breast cancer. Clin Exp Metastasis 30: 951-956, 2013.

14. Sperduto PW, Kased N, Roberge D, Chao ST, Shanley R, Luo X, Sneed PK, Suh J, Weil RJ, Jensen AW, et al: The effect of tumor subtype on the time from primary diagnosis to development of brain metastases and survival in patients with breast cancer. J Neurooncol 112: 467-472, 2013.

15. Hammond ME, Hayes DF, Wolff AC, Mangu PB and Temin S: American society of clinical oncology/college of american pathologists guideline recommendations for immunohistochemical testing of estrogen and progesterone receptors in breast cancer. J Oncol Pract 6: 195-197, 2010.

16. Wolff AC, Hammond ME, Hicks DG, Dowsett M, McShane LM, Allison KH, Allred DC, Bartlett JM, Bilous M, Fitzgibbons P, et al: Recommendations for human epidermal growth factor receptor 2 testing in breast cancer: American Society of Clinical Oncology/College of American Pathologists clinical practice guideline update. J Clin Oncol 31: 3997-4013, 2013.

17. Kimbung S, Loman $\mathrm{N}$ and Hedenfalk I: Clinical and molecular complexity of breast cancer metastases. Semin Cancer Biol 35: $85-95,2015$.

18. Chaffer CL and Weinberg RA: A perspective on cancer cell metastasis. Science 331: 1559-1564, 2011

19. Goldhirsch A, Wood WC, Coates AS, Gelber RD, Thurlimann B and Senn HJ; Panel members: Strategies for subtypes-dealing with the diversity of breast cancer: Highlights of the St. Gallen international expert consensus on the primary therapy of early breast cancer 2011. Ann Onco 22: 1736-1747, 2011.

20. Ongaro E, Gerratana L, Cinausero M, Pelizzari G, Poletto E, Giangreco M, Andreetta C, Pizzolitto S, Di Loreto C Minisini AM, et al: Comparison of primary breast cancer and paired metastases: Biomarkers discordance influence on outcome and therapy. Future Oncol 14: 849-859, 2018.

21. Killelea BK, Gallagher EJ, Feldman SM, Port E, King T, Boolbol SK, Franco R, Fei K, Le Roith D and Bickell NA: The effect of modifiable risk factors on breast cancer aggressiveness among black and white women. Am J Surg 218: 689-694, 2019.

22. Fujii K, Watanabe R, Ando T, Kousaka J, Mouri Y, Yoshida M, Imai T, Nakano $\mathrm{S}$ and Fukutomi T: Alterations in three biomarkers (estrogen receptor, progesterone receptor and human epidermal growth factor 2) and the Ki67 index between primary and metastatic breast cancer lesions. Biomed Rep 7: 535-542, 2017.

23. Soni A, Ren Z, Hameed O, Chanda D, Morgan CJ, Siegal GP and Wei S: Breast cancer subtypes predispose the site of distant metastases. Am J Clin Pathol 143: 471-478, 2015.

24. Schmalhofer O, Brabletz $\mathrm{S}$ and Brabletz T: E-cadherin, beta-catenin, and ZEB1 in malignant progression of cancer. Cancer Metastasis Rev 28: 151-166, 2009.
25. Zhang Y, He W and Zhang S: Seeking for correlative genes and signaling pathways with bone metastasis from breast cancer by integrated analysis. Front Oncol 9: 138, 2019.

26. Jones DH, Nakashima T, Sanchez OH, Kozieradzki I, Komarova SV, Sarosi I, Morony S, Rubin E, Sarao R, Hojilla CV, et al: Regulation of cancer cell migration and bone metastasis by RANKL. Nature 440: 692-696, 2006.

27. Acharya S, Yao J, Li P, Zhang C, Lowery FJ, Zhang Q, Guo H, Qu J, Yang F, Wistuba II, et al: Sphingosine-kinase-1 signaling promotes metastasis of triple-negative breast cancer. Cancer Res 79: 4211-4226, 2019.

28. Li YM, Pan Y, Wei Y, Cheng X, Zhou BP, Tan M, Zhou X, Xia W, Hortobagyi GN, Yu D and Hung MC: Upregulation of CXCR4 is essential for HER2-mediated tumor metastasis. Cancer Cell 6: 459-469, 2004.

29. Wu W, Chen J, Ye W, Li X and Zhang J: Fatty liver decreases the risk of liver metastasis in patients with breast cancer: A two-center cohort study. Breast Cancer Res Treat 166: 289-297, 2017.

30. Park HS, Kim S, Kim K, Yoo H, Chae BJ, Bae JS, Song BJ and Jung SS: Pattern of distant recurrence according to the molecular subtypes in Korean women with breast cancer. World J Surg Oncol 10: 4, 2012

31. Carvalho ST, Stiepcich MM, Fregnani JH, Nonogaki S, Rocha R and Soares FA: Evaluation of prognostic factors in stage IIA breast tumors and their correlation with mortality risk. Clinics (Sao Paulo) 66: 607-612, 2011.

32. Zhang C, Wang S, Israel HP, Yan SX, Horowitz DP, Crockford S, Gidea-Addeo D, Clifford Chao KS, Kalinsky K and Connolly EP: Higher locoregional recurrence rate for triple-negative breast cancer following neoadjuvant chemotherapy, surgery and radiotherapy. Springer Plus 4: 386, 2015.

33. Giuli MV, Giuliani E, Screpanti I, Bellavia D and Checquolo S: Notch signaling activation as a hallmark for triple-negative breast cancer subtype. J Oncol 2019: 8707053, 2019.

34. Press DJ, Miller ME, Liederbach E, Yao K and Huo D: De novo metastasis in breast cancer: Occurrence and overall survival stratified by molecular subtype. Clin Exp Metastasis 34: 457-465, 2017.

35. Bonotto M, Gerratana L, Poletto E, Driol P, Giangreco M, Russo S, Minisini AM, Andreetta C, Mansutti M, Pisa FE, et al: Measures of outcome in metastatic breast cancer: Insights from a real-world scenario. Oncologist 19: 608-615, 2014.

This work is licensed under a Creative Commons Attribution-NonCommercial-NoDerivatives 4.0 International (CC BY-NC-ND 4.0) License. 